\title{
Dynamical systems, shape theory and the Conley index
}

\author{
JOEL W. ROBBIN AND DIETMAR SALAMON \\ Department of Mathematics, University of Wisconsin, Madison, Wisconsin 53706, \\ USA; Mathematics Institute, University of Warwick, Coventry CV4 7AL, UK
}

Abstract. The Conley index of an isolated invariant set is defined only for flows; we construct an analogue called the 'shape index' for discrete dynamical systems. It is the shape of the one-point compactification of the unstable manifold of the isolated invariant set in a certain topology which we call its 'intrinsic' topology (to distinguish it from the 'extrinsic' topology which it inherits from the ambient space). Like the Conley index, it is invariant under continuation. A key point is the construction of a certain 'index category' associated with the isolated invariant set; this construction works equally well for flows or discrete time systems, and its properties imply the basic properties of both the Conley index and the shape index.

\section{Introduction}

The homotopy index developed over the years by C. C. Conley and his students has been justly termed the Conley index. Although virtually all of Conley's students had some part in the development of the theory of this index, the version closest to the one we present here first appeared in the thesis of Kurland (published as [6]). Recent expositions appear in [1], [2], [9] and [11].

The basic theory of the Conley index may be summarized as follows:

(1) The Conley index of an isolated invariant set of a flow is independent of the index pair used to define it.

(2) The Conley index is invariant under continuation.

(Definitions will be given in the sequel.) We shall prove the following analogue which works equally well for discrete time dynamical systems and flows:

(1) The intrinsic topology of the unstable manifold of an isolated invariant set of a dynamical system is independent of the index pair used to define it.

(2) The shape of the one-point compactification of this unstable manifold is invariant under continuation.

The main ingredient of the proof is the construction of maps

$$
f_{\beta \alpha}^{t}: N_{\alpha} / L_{\alpha} \rightarrow N_{\beta} / L_{\beta}
$$

associated to pairs of index pairs $\left(N_{\alpha}, L_{\alpha}\right)$ and $\left(N_{\beta}, L_{\beta}\right)$ and non-negative times $t$, and satisfying the identity

$$
f_{\gamma \beta}^{s} \circ f_{\beta \alpha}^{t}=f_{\gamma \alpha}^{s+t}
$$


This determines a category which we call the index category of the invariant set.

The idea of the Conley index goes back to Morse and Smale. Morse showed that the topology of the sublevel sets of a smooth function changed when the level moved through a critical level and thereby proved his inequalities relating the Betti numbers of the manifold and the indices of the critical points. Smale [10] defined certain dynamical systems (axiom A and no cycles), showed they admitted 'filtrations' analogous to the filtration of a manifold by the sublevel sets of a Morse function and thereby extended Morse theory from gradient dynamical systems to a vastly more general class. The monograph [4] summarizes some of the fruits of this line of thinking.

Conley and his students made the following two essential contributions to all this. Firstly, they showed that the theory could be applied to any isolated invariant set, not just hyperbolic ones. Secondly, they 'localized' the theory in the sense that the index pair $(N, L)$ need not consist of adjacent levels of a filtration, i.e. one need not assume that orbits which leave a neighbourhood of the invariant set never return. (Invariant sets which do have this property Conley termed 'Morse sets'.) Conley also suggested to us the idea that the analogue for discrete dynamical systems of the cohomology index should be the direct limit.

\section{Notation and terminology}

Throughout $\boldsymbol{M}$ denotes a compact smooth manifold (although many of our arguments work on a locally compact metric space). By a dynamical system on $M$ we mean a smooth homomorphism of groups

$$
T \rightarrow \operatorname{Diff}(M): t \rightarrow f^{t}
$$

from the additive group $T$ into the group $\operatorname{Diff}(M)$ of diffeomorphisms from $M$ onto itself. We consider two cases simultaneously: discrete time $T=Z$ so that $f \in \operatorname{Diff}(M)$ and $f^{t}$ denotes the $t$ th iterate of $f$, and continuous time $T=\mathbf{R}$ so that $f$ is a flow. In the continuous time case the smoothness hypothesis means that the evaluation map $\mathrm{R} \times M \rightarrow M:(t, x) \mapsto f^{t}(x)$ is smooth $\left(C^{1}\right)$. In the continuous time case we call the vector field $v \in \mathscr{L}(M)$ defined by

$$
v(x)=\left.\frac{d}{d t} f^{t}(x)\right|_{t=0} \in T_{x} M
$$

the infinitesimal generator of $f$.

If $J \subset T$ and $X \subset M$, we write $f^{\prime}(X)$ where some authors write $X \cdot J$ :

$$
f^{J}(X)=\left\{f^{\prime}(x): t \in J, x \in X\right\} .
$$

When $x \in M$, define $f^{J}(x)=f^{J}(\{x\})$. In the discrete time case $(T=Z)$, if $J \subset \mathbf{R}$, the notation is understood as if $J$ were replaced by $J \cap Z: f^{J}(X)=f^{J \cap Z}(X)$; thus $f^{[3 / 2,3]}(x)=\left\{f^{2}(x), f^{3}(x)\right\}$ when $T=Z$.

We denote by $T^{+}$the non-negative elements of $T$; thus $\mathbf{R}^{+}=[0, \infty)$ and $\mathbf{Z}^{+}=\mathbf{Z} \cap$ $\mathbf{R}^{+}=\mathbf{N}$, the natural numbers.

If $(N, L)$ is a compact pair in $M$ (i.e. if $L \subset N \subset M$ and $L$ and $N$ are compact), then we denote by $N / L$ the space which results from $N$ when the elements of $L$ 
are identified to a point. More precisely,

$$
N / L=N \backslash L \cup\{*\},
$$

where the topology is chosen so that the projection map $N \rightarrow N / L$ (which is the identity on $N \backslash L$ and sends $L$ to $*$ ) is continuous and restricts to a homeomorphism from $N \backslash L$ onto $N / L \backslash\{*\}$. We shall not distinguish between the subset $N \backslash L$ of $M$ and the subset $N / L \backslash\{*\}$ of $N / L$. Note that $N / \varnothing$ is obtained from $N$ by adjoining a single (isolated) point: $N / \varnothing=N \cup\{*\}$.

We denote by $\mathrm{cl}(V)$, int $(V)$ and $\partial(V)$ respectively the closure, interior and (set-theoretic) boundary of a subset $V$ of $M$. The reader should take care to distinguish two similar notations with different meanings: $N \backslash L$ (set-theoretic difference) and $N / L$ (smash $L$ to the base point). The notation

$$
f \sim g
$$

shall mean that the maps $f$ and $g$ are homotopic and $[X]$ denotes the homotopy type of the space $X$, i.e.

$$
[X]=[Y]
$$

if and only if $X$ and $Y$ are homotopy equivalent.

\section{An overview of shape theory}

Shape theory was invented by Borsuk; our main reference is [7]. An older reference explaining inverse and direct systems and their limits is [3]. The definitions (given below) are arranged so that the following theorems are true.

THEOREM 2.1. Every compact metric space $X$ is homeomorphic to an inverse limit of polyhedra lim $(P, p)$.

THEOREM 2.2. The inverse system of polyhedra converging to $X$ is unique in the following sense. If $(P, p)$ and $(Q, q)$ are inverse systems whose spaces are polyhedra (or even homotopy polyhedra) and if $\lim (P, p)$ and $\lim (Q, q)$ are homeomorphic (or even only homotopy equivalent), then the inverse systems $(P, p)$ and $(Q, q)$ are shape equivalent.

THEOREM 2.3. Let $F$ be an algebraic functor as described below. Then any shape equivalence between $(P, p)$ and $(Q, q)$ induces an isomorphism between $\lim (F(P), F(p))$ and $\lim (F(Q), F(q))$, where $\lim$ denotes the inverse limit if the functor $F$ is covariant and the direct limit if it is contravariant.

Definition 2.4. We say that compact metric spaces $X$ and $Y$ have the same shape and write

$$
\operatorname{Sh}(X)=\operatorname{Sh}(Y)
$$

if and only if there are inverse systems of polyhedra $(P, p)$ and $(Q, q)$ where $X$ is homeomorphic to $\lim (P, p), Y$ is homeomorphic to $\lim (Q, q)$, with $(P, p)$ and $(Q, q)$ shape equivalent.

Theorems 2.1 and 2.2 assure that this definition is meaningful and theorem 2.3 says that the usual functors of algebraic topology (singular homology, singular 
cohomology, homotopy, etc.) extend in a natural (from the point of view of shape theory) way from the category of homotopy polyhedra to the category of compact metric spaces. For example, one way to express $X$ as an inverse limit of polyhedra is via the so-called $\hat{C}$ ech system [7, p 327] of nerves of open coverings of $X$. The limit of the simplicial homology of the Ĉech system is precisely Ĉech homology (usual definition), so that the extension of homology given by shape theory agrees with Ĉech homology [7, p 122].

COROLlary 2.5. Two homotopy polyhedra have the same shape if and only if they have the same homotopy type.

Corollary 2.6. Suppose $(P, p)$ and $(P, q)$ are inverse systems over the same directed set having the same spaces but possibly different bonding morphisms. If $p_{a b}$ is homotopic to $q_{a b}$ for all $a, b$ with $a \leq b$, then the inverse limits have the same shape:

$$
\operatorname{Sh}(\lim (P, p))=\operatorname{Sh}(\lim (P, q)) \text {. }
$$

COROLlaRY 2.7. If $X=\lim (P, p)$ is an inverse limit of homotopy polyhedra and each bonding morphism $p_{a b}$ is a homotopy equivalence, then $X$ has the same shape as each of the approximating spaces: $\operatorname{Sh}(X)=\operatorname{Sh}\left(P_{a}\right)$ for all $a$.

Here are the relevant definitions. First, by theorem 7 (p 319) and theorem 8 (p 320) of [7], a compact topological space has the homotopy type of a compact polyhedron if and only if it has the homotopy type of a compact CW-complex if and only if it has the homotopy type of a compact ANR; we call such a space a homotopy polyhedron.

By an inverse system over a directed set $A$ we mean a pair $(P, p)$, where $P$ is a function which assigns to each $a \in A$ a space $P_{a}$ and $p$ is a function which assigns to $a, b \in A$ with $a \leq b$ a morphism $p_{a b}: P_{b} \rightarrow P_{a}$ such that $p_{a b}{ }^{\circ} p_{b c}=p_{a c}$ for $a \leq b \leq c$ and $p_{a a}=\mathrm{id}$, the identity map of $P_{a}$. The spaces $P_{a}$ are called the spaces of the system, while the morphisms $p_{a b}$ are called the bonding morphisms. Let $(Q, q)$ be another inverse system over a directed set $B$. An indexed family of morphisms

$$
(f, \phi):(P, p) \rightarrow(Q, q)
$$

consists of an order-preserving map $\phi: B \rightarrow A$ and a function $f$ which assigns to each $b \in B$ a map $f_{b}: P_{\phi(b)} \rightarrow Q_{b}$. The composition of two families $(g, \psi) \circ(f, \phi)=$ $(h, \phi \circ \psi)$ is defined by $h_{c}=g_{c} \circ f_{\psi(c)}$. We call a family:

commuting iff for all $b, c \in B$ with $b \leq c$ we have

$$
f_{b} \circ p_{\phi(b) \phi(c)}=q_{b c} \circ f_{c}
$$

ultimately commuting iff for all $b, c \in B$ with $b \leq c$ there exists $a \in A$ with $a \geq \phi(b), \phi(c)$ and

$$
f_{b} \circ p_{\phi(b) a}=q_{b c} \circ f_{c} \circ p_{\phi(c) a} ;
$$

homotopy commuting iff for all $b, c \in B$ with $b \leq c$ we have

$$
f_{b} \circ p_{\phi(b) \phi(c)} \sim q_{b c} \circ f_{c} ;
$$

ultimately homotopy commuting iff for all $b, c \in B$ with $b \leq c$ there exists $a \in A$ with $a \geq \phi(b), \phi(c)$ and

$$
f_{b} \circ p_{\phi(b) a} \sim q_{b c} \circ f_{c} \circ p_{\phi(c) a} .
$$


Given two families $(f, \phi),(g, \psi):(P, p) \rightarrow(Q, q)$, call them:

ultimately equal iff for all $b \in B$ there exists $a \in A$ with $a \geq \phi(b), \psi(b)$ and

$$
f_{b} \circ p_{\phi(b) a}=g_{b} \circ p_{\phi(b) a} ;
$$

ultimately homotopic iff for all $b \in B$ there exists $a \in A$ with $a \geq \phi(b), \psi(b)$ and

$$
f_{b} \circ p_{\phi(b) a} \sim g_{b} \circ p_{\psi(b) a} .
$$

Two inverse systems $(P, p)$ and $(Q, q)$ are called shape equivalent if there exist ultimately homotopy commuting families $(f, \phi):(P, p) \rightarrow(Q, q)$ and $(g, \psi):(Q, q) \rightarrow$ $(P, p)$ such that the composition $(g, \psi) \circ(f, \phi)$ is ultimately homotopic to the identity family of $(P, p)$ and the composition $(f, \phi) \circ(g, \psi)$ is ultimately homotopic to the identity family of $(Q, q)$; the family $(f, \phi)$ is then called a shape equivalence.

By an algebraic functor we mean one defined on the homotopy category of homotopy polyhedra into a category which admits inverse limits if the functor is covariant and direct limits if the functor is contravariant. Any functor into the category of groups is an example.

We have now defined all the terminology needed to understand the statements of 2.1-2.7 (except perhaps that which can be found in [3]) and proceed to guide the reader to the proofs. The reader is invited to test his/her understanding of the definitions by showing that the Warsaw circle of [7] ( $p$ xiii and example 4 on $p$ 67) has the shape of the circle.

A proof of theorem 2.1 is given on $\mathrm{p} 61$ of [7]. Theorem 2.2 comes from theorem 9 on p 65 and remark 2 on p 19. For theorem 3 see theorem 2 on p 122, remark 2 on $\mathrm{p} 124$ and theorem 4 on $\mathrm{p} 128$.

Corollary 2.5 is a triviality. Any space is the inverse limit of a trivial inverse system having a directed set of one element, viz. the space itself. A shape equivalence between two such trivial inverse systems is the same thing as a homotopy equivalence. Corollary 2.6 is also a triviality; the identity map is a shape equivalence.

For corollary 2.7 we must construct a shape equivalence between the inverse system $(P, p)$ over $A$ and the trivial inverse system $(Q, i)$ over $\{1\}$, where $Q_{1}=P_{a}$ and $i_{11}=\mathrm{id}$, the identity map of $P_{a}$. Let $\iota:\{1\} \rightarrow A$ be given by $\iota(1)=a$ and $\pi: A \rightarrow\{1\}$ be given by $\pi(b)=1$ for all $b$. Define $(f, \iota):(P, p) \rightarrow(Q, i)$ by $f_{1}=$ id and $(g, \pi):(Q, i) \rightarrow(P, p)$ by $g_{b}=p_{b c} g_{c a}$, where $g_{c a}: P_{a} \rightarrow P_{c}$ is a homotopy inverse to $p_{a c}$ and $c=c(b)$ is any index larger than $a$ and $b$. (The homotopy class of $g_{b}$ is independent of the choice of $c$ as $d \geq c$ implies $p_{b c}{ }^{\circ} g_{c a} \sim p_{b c} \circ p_{c d} \circ g_{d c} \circ g_{c a} \sim$ $\left.p_{b d} \circ g_{d a}.\right)$ Then $(f, \iota) \circ(g, \pi)$ is the identity of $(Q, i)$ (which is a commuting family) while $(g, \pi) \circ(f, \iota)=(g, \alpha)$, where $\alpha: A \rightarrow A$ is given by $\alpha(b)=a$ for all $b .(g, \alpha)$ is an ultimately commuting family as $b \leq d$ implies $p_{b d} \circ g_{d} \sim p_{b d} \circ p_{d e} \circ g_{e a}=p_{b e} \circ g_{e a} \sim$ $g_{b}$ for large $e$. To show that $(g, \alpha)$ is ultimately homotopic to the identity, we must show that for every $b \in A$ there exists $d \in A$ with $d \geq b$ and $d \geq a=\alpha(b)$ and $g_{b} \circ p_{a d} \sim p_{b d} ; d=c$ works.

LEMMA 2.8. Suppose $\Phi$ and $\Psi$ are smooth real-valued functions on $M$, that 0 is a regular value for both $\Phi$ and $\Psi$, and that $\Phi^{-1}(0)$ and $\Psi^{-1}(0)$ intersect transversally. Let

$$
N=\Phi^{-1}([0, \infty)) \cap \Psi^{-1}([0, \infty))
$$


and

$$
L=N \cap \Phi^{-1}(0)
$$

(Thus $N$ is a manifold with corners.) Then the space $N / L$ is a homotopy polyhedron.

Proof. In standard references on Morse theory such as [8] or [5] it is proved that on a manifold with a boundary one can always find a Morse function having the boundary as a regular level and that one can use that Morse function to extend a cellular decomposition of the boundary to the whole manifold. Using this fact, first decompose $\Phi^{-1}(0) \cap \Psi^{-1}(0)$ (no boundary), then decompose $N \cap \Phi^{-1}(0)$ and $N \cap$ $\Psi^{-1}(0)$ (boundary is $\left.\Phi^{-1}(0) \cap \Psi^{-1}(0)\right)$, extend this decomposition to a 'collar' of $\partial N$ of form $(\Phi \Psi)^{-1}([0, \varepsilon])$ (product with an interval) and finally extend to $N$ (boundary is $(\Phi \Psi)^{-1}(\varepsilon)$ ). Now we have made $N$ into a $C W$-complex with $L$ a subcomplex. Hence $N / L$ is a $C W$-complex.

\section{Isolated invariant sets and continuation}

Fix a dynamical system $f$ on $M$. Given any set $V$, denote by $I(V, f)$ the maximal invariant set inside of $V$ :

$$
I(V, f)=\bigcap_{i \in T} f^{t}(V) .
$$

An isolating neighbourhood for $f$ is a set $V$ such that the maximal invariant set in its closure is contained in its interior:

$$
I(\mathrm{cl}(V), f) \subset \operatorname{int}(V) \text {; }
$$

an isolated invariant set for $f$ is a set $S$ of form

$$
S=I(\operatorname{cl}(V), f)
$$

for some isolating neighbourhood $V$ (one then says that $V$ is an isolating neighbourhood for $(S, f))$.

Proposition 3.1. Let $V_{1}$ and $V_{2}$ be isolating neighbourhoods for the same isolated invariant set $S$ of $f$. Then

$$
\bigcap_{-t_{0} \leq t \leq t_{0}} f^{t}\left(\mathrm{cl}\left(V_{1}\right)\right) \subset \operatorname{int}\left(V_{2}\right)
$$

for sufficiently large $t_{0}$.

Proof. Compactness.

COROLlaRY 3.2. For all dynamical systems $g$ sufficiently near $f$ (in the $C^{0}$ topology) we have

$$
I\left(V_{1}, g\right)=I\left(V_{2}, g\right) .
$$

COROLLARY 3.3. The set of all dynamical systems having a given set $V$ as an isolating neighbourhood is open in the space of all dynamical systems on $M\left(C^{0}\right.$ topology).

Let $J=[0,1]$ denote the unit interval. A continuation of isolated invariant sets on $M$ is a collection

$$
\left\{\left(S_{\lambda}, f_{\lambda}\right): \lambda \in J\right\}
$$


of pairs indexed by $J$ such that the dynamical system $F$ on $M \times I$ given by

$$
F^{t}(x, \lambda)=\left(f_{\lambda}^{t}(x), \lambda\right)
$$

(for $t \in T, x \in M, \lambda \in \dot{I}$ ) is smooth and the set $\Sigma \subset M \times J$ defined by

$$
\Sigma=\left\{(x, \lambda): x \in S_{\lambda}\right\}
$$

is an isolated invariant set for $F$; one calls this a continuation from $\left(S_{0}, f_{0}\right)$ to $\left(S_{1}, f_{1}\right)$. The relation 'there is a continuation from $\left(S_{0}, f_{0}\right)$ to $\left(S_{1}, f_{1}\right)$ ' is an equivalence relation on the space of all pairs $(S, f)$ with $S$ an isolated invariant set of $f$. Let $W=\bigcup_{\lambda} V_{\lambda} \times\{\lambda\}$ be an isolating neighbourhood for $(\Sigma, F)$. Then $V_{\lambda}$ is an isolating neighbourhood for $f_{\lambda}$ and

$$
S_{\lambda}=I\left(V_{\lambda}, f_{\lambda}\right),
$$

so each $S_{\lambda}$ is an isolated invariant set for $f_{\lambda}$. Since each $V_{\lambda}$ is also an isolating neighbourhood for $f_{\mu}$ for $\mu$ sufficiently near $\lambda$, we have:

Proposition 3.4. A continuation is 'locally trivial' in the sense that there is a covering of $J$ by open intervals $J_{j}(j=1,2, \ldots, n)$ and a collection $V_{j}(j=1,2, \ldots, n)$ of subsets of $M$ such that for $\lambda \in J_{j}$ we have that $V_{j}$ is an isolating neighbourhood for $f_{\lambda}$ and

$$
S_{\lambda}=I\left(V_{j}, f_{\lambda}\right) \text {. }
$$

The role of continuations in the theory of dynamical systems is analogous to the role of homotopies in topology. Of course, in applications one constructs the continuation by constructing the isolating neighbourhood $W \subset M \times I$.

4. The induced semidynamical system of a pair

Definition 4.1. Given a compact pair $(N, L)$ in $M$ and $t \in T^{+}$, define a map

$$
f_{\#}^{t}: N / L \rightarrow N / L
$$

by

$$
f_{\#}^{t}(x)= \begin{cases}f^{t}(x) & \text { if } f^{[0, t]}(x) \subset N \backslash L . \\ * & \text { otherwise. }\end{cases}
$$

Note that if $f_{\# \#}^{t}(x)=*$ for some $t$, then this continues to hold for all larger $t$. Also note that the family of maps $\left\{f_{\#}^{t}\right\}$ satisfies the semigroup property

$$
f_{\#}^{0}=\mathrm{id}, \quad f_{\#}^{t+s}=f_{\#}^{t} \circ f_{\#}^{s}
$$

for $t, s \in T^{+}$, where id denotes the identity map of $N / L$. We call $t \mapsto f_{\#}^{t}$ the semidynamical system induced by $f$ on the space $N / L$.

TheOrem 4.2. Assume the continuous case: $T=\mathbf{R}$. Then the evaluation map

$$
\mathbf{R}^{+} \times N / L \rightarrow N / L:(t, x) \mapsto f_{\#}^{t}(x)
$$

is continuous if and only if:

(i) $L$ is positively invariant relative to $N$, i.e.

$$
x \in L, \quad f^{[0, r]}(x) \subset N \Rightarrow f^{[0, t]}(x) \subset L .
$$

(ii) Every orbit which exits $N$ goes through $L$ first:

$$
x \in N, \quad f^{[0, \infty)}(x) \not \subset N \Rightarrow \exists t \geq 0 \text { with } f^{[0, t]} \subset N \text { and } f^{\prime}(x) \in L .
$$


Proof. First we assume that (i) fails and show that $f_{\text {\# }}$ fails to be continuous at some $(t, *)$. Assume that $x \in L$ and $f^{[0, t]}(x) \subset N$ but $f^{t}(x) \notin L$. On replacing $x$ by $f^{s_{0}}(x)$ and $t$ by $t-s_{0}$, where $s_{0}=\sup \left\{s \in[0, t]: f^{s}(x) \in L\right\}$, we may assume without loss of generality that $f^{s}(x) \in N \backslash L$ for $0<s \leq t$. Let $x_{n}=f^{1 / n}(x)$ and $t_{n}=t-1 / n$. Then $\left(t_{n}, x_{n}\right)$ converges to $(t, *)$ in $\mathbf{R}^{+} \times N / L$ but $f_{\#}^{t_{n}}\left(x_{n}\right)=f^{t}(x) \neq *=f_{\#}^{t}(*)$; i.e. $f_{\#}^{t_{n}}\left(x_{n}\right)$ does not converge to $f_{*}^{t}(*)$.

Next we assume that (ii) fails and show that $f_{\#}$ fails to be continuous at some $(s, x)$ with $x \in N \backslash L$. Assume that $x \in N$ and $f^{[0, \infty)}(x) \not \subset N$ but that the requisite $t$ fails to exist. Let $s=\sup \left\{t \in[0, \infty): f^{[0, t]}(x) \subset N\right\}$ so that there is a sequence $s_{n}$ converging to $s$ with $f^{s_{n}}(x) \notin N$; i.e. $f_{\#}^{s_{n}}(x)=*$. But as $N$ is closed, $f^{[0, s]}(x) \subset N$ anà so by hypothesis there can be no $t \in[0, s]$ with $f^{t}(x) \in L$; thus $f^{[0, s]} \subset N \backslash L$, i.e. $f_{*}^{s}(x)=f^{s}(x) \neq *$. Thus $f_{\#}$ is not continuous at $(s, x) \in \mathbf{R}^{+} \times(N \backslash L) \subset \mathbf{R}^{+} \times(N / L)$ as required.

Now assume (i) and (ii), but that $f_{\#}$ is not continuous, i.e. that there is a sequence $\left\{x_{n}\right\} \subset N / L$ converging to $x \in N$ (by compactness) and a sequence $\left\{t_{n}\right\}$ converging to $t \in \mathbf{R}^{+}$with $f_{*}^{t_{n}}\left(x_{n}\right)$ not converging to $f_{\#}^{t}(x)$. We must derive a contradiction. We distinguish two cases: $f_{\#}^{\prime}(x)=*$ and $f_{\# *}^{\prime}(x)=f^{t}(x) \in N \backslash L$.

First consider the case $f_{\#}^{t}(x)=*$. Then passing to a subsequence, we may assume $f_{n}^{t}\left(x_{n}\right)=f^{t_{n}}\left(x_{n}\right) \in N \backslash L$ and that $f^{t}(x) \notin L$ (else we would have convergence). But $f^{\left[0, t_{n}\right]}\left(x_{n}\right) \subset N \backslash L \subset N$ so $f^{[0, t]}(x) \subset N$ as $N$ is closed. Since $f_{\#}^{t}(x)=*$, we must have $f^{[0, t]}(x) \cap L \neq \varnothing$, which together with $f^{\prime}(x) \notin L$ contradicts (i).

Now consider the case $f_{\#}^{t}(x)=f^{t}(x) \in N \backslash L$. Then passing to a subsequence and using the continuity of $f$, we may assume that $f_{\#}^{t_{n}}\left(x_{n}\right)=*$ for all $n$ so $f^{\left[0, t_{n}\right]}\left(x_{n}\right) \not \subset N \backslash L$. Either $f^{\left[0, t_{n}\right]}\left(x_{n}\right) \not \subset N$ or $f^{\left[0, t_{n}\right]}\left(x_{n}\right) \cap L \neq \varnothing$; in either case choose $s_{n} \in\left[0, t_{n}\right]$ so that $f^{s}\left(x_{n}\right) \in L$ (in the former case we use (ii)). The points $f^{s_{n}}\left(x_{n}\right)$ accumulate at a point $f^{s}(x) \in L$, which contradicts the assumption that $f_{\#}^{t}(x)=f^{\prime}(x) \in N \backslash L$ (for that would require $\left.f^{[0, t]}(x) \subset N \backslash L\right)$. This completes the proof.

THEOREM 4.3. Assume the discrete case: $T=Z$. Then $f_{*}$ is continuous if and only if:

(A0) Every $x_{0} \in L \cap f^{-1}(N \backslash L)$ has a neighbourhood $A_{0}$ in $M$ with $f\left(A_{0} \cap N \backslash L\right) \subset$ $M \backslash N$. (This means that whenever $x_{0} \in L$ but $f\left(x_{0}\right) \in N \backslash L$ we have $f_{*}(x)=*$ for all $x \in N \backslash L$ sufficiently near $x_{0}$.)

(B0) Every $x_{0} \in(N \backslash L) \cap f^{-1}((\partial N) \backslash L)$ has a neighbourhood $B_{0}$ in $M$ with $f\left(B_{0} \cap\right.$ $N \backslash L) \subset N$. (This means that whenever $x_{0} \in N \backslash L$ and $f_{\#}\left(x_{0}\right)=f\left(x_{0}\right) \in N \backslash L$ then $f_{\#}(x)=f(x) \in N \backslash L$ for all $x$ sufficiently near $x_{0}$.)

Proof. Assume condition (A0) fails. We will show that $f_{\#}$ is not continuous at *. We have $x_{0} \in L$ with $f\left(x_{0}\right) \in N \backslash L$, and no $A_{0}$ exists as in (A0); thus there is a sequence $x_{n} \in N \backslash L$ converging to $x_{0}$ with $f\left(x_{n}\right) \in N$. Since $L$ is closed and $f\left(x_{0}\right) \in N \backslash L$, we even have $f\left(x_{n}\right) \in N \backslash L$. But then $f_{\#}\left(x_{n}\right)=f\left(x_{n}\right)$ converges to $f\left(x_{0}\right) \in N \backslash L$ while $x_{n}$ converges to $*$ in $N \backslash L$. Thus $f_{\#}$ is not continuous.

Next assume condition (B0) fails for some $x_{0} \in N \backslash L$. We will show that $f_{*}$ is not continuous at $x_{0}$. Then $x_{0} \in N \backslash L$ and $f\left(x_{0}\right) \in N \backslash L$, but since no $B_{0}$ exists as in (B0), there is a sequence $x_{n} \in N \backslash L$ converging to $x_{0}$ with $f\left(x_{n}\right) \notin N$. Then $f_{*}\left(x_{n}\right)=*$ does not converge in $N / L$ to $f_{\#}\left(x_{0}\right)=f\left(x_{0}\right) \in N \backslash L$ so that $f_{\#}$ is not continuous. 
Now we assume (A0) and (B0) and prove that $f_{\#}$ is continuous on $N / L$. There are four steps.

$f_{\#}$ is continuous at $x_{0} \in(N \backslash L) \cap f^{-1}(L)$. For $x$ near $x_{0}, f(x)$ is near $f\left(x_{0}\right) \in L$ and so $f_{\#}(x)$ is near $*$.

$f_{\#}$ is continuous at $x_{0} \in(N \backslash L) \cap f^{-1}(M \backslash N)$ since $f_{\#}(x)=*$ for $x$ near $x_{0}$.

$f_{*}$ is continuous at $x_{0} \in(N \backslash L) \cap f^{-1}(N \backslash L)$ since (B0) ensures that $f_{*}(x)=f(x) \in$ $N \backslash L$ for $x$ near $x_{0}$.

$f_{*}$ is continuous at $*$. We assume not, and derive a contradiction. There is a sequence $x_{n} \in N \backslash L$ converging to $*$ in $N / L$ with $f_{\#}\left(x_{n}\right)$ converging to $y \in N \backslash L$. Pass to a subsequence so that $x_{n}$ converges to $x_{0} \in L$. Then $f\left(x_{n}\right)=f_{\#}\left(x_{n}\right)$ converges to $f\left(x_{0}\right)=y \in N \backslash L$. This contradicts the existence of $A_{0}$ as in (A0).

This completes the proof of theorem 4.3.

The analogues of the necessary and sufficient conditions of theorem 4.2 are only sufficient in the discrete case. These conditions are positive invariance of $L$ in $N$ : $f(L) \cap N \subset L$ (which says that if $x \in L$ and $f(x) \in N$ then $f(x) \in L$ ) and the exit set property: $N \cap f^{-1}(M \backslash N) \subset L$ (which says that if $f(x)$ is the first point for which some forward orbit originating in $N$ leaves $N$, then $x \in L$ ).

Corollary 4.4. Assume the discrete case: $T=\mathbf{Z}$. Assume

$$
f(L) \cap N \subset L
$$

and

$$
N \cap f^{-1}(M \backslash N) \subset L
$$

Then $f_{\#}$ is continuous.

Proof. $L \cap f^{-1}(N \backslash L)$ is empty so condition (A0) holds vacuously. Also $N \cap$ $f^{-1}(M \backslash N) \subset L$ implies $f(N) \backslash N \subset f(L)$ which implies $f(N \backslash L) \subset N$ so that $(N \backslash L) \cap$ $f^{-1}(N \backslash L) \subset B_{0}$ and $f\left(B_{0} \cap N \backslash L\right) \subset N$ with $B_{0}=M \backslash L$. This proves condition (B0).

Theorem 4.5. Assume the discrete case: $T=Z$. Assume $N \subset M$ is closed and

$$
(\partial N) \cap f^{-1}(N) \cap f(N)=\varnothing .
$$

Define

$$
L_{0}=f(N) \cap(\partial N)
$$

and

$$
L_{1}=\operatorname{cl}\left(N \backslash f^{-1}(N)\right) .
$$

Then $L_{0} \subset L_{1}$ and $f_{\#}: N / L \rightarrow N / L$ is continuous for any closed subset $L$ of $N$ satisfying $L_{0} \subset L \subset L_{1}$.

Proof. Our hypothesis can be rewritten as

$$
f(N) \cap(\partial N) \subset M \backslash f^{-1}(N),
$$

so intersecting both sides with $N$ gives $L_{0} \subset L_{1}$, Clearly

$$
f\left(L_{1}\right) \cap N \subset L_{0}
$$

so that for $L_{0} \subset L \subset L_{1}$ we have $f(L) \cap N \subset L$ whence $L \cap f^{-1}(N \backslash L)=\varnothing$, so condition (A0) holds vacuously. 
Condition (B0) also holds vacuously when $L_{0} \subset L$. This is because $N \backslash L \subset N$ and $(\partial N) \backslash L \subset(\partial N) \backslash L_{0}=(\partial N) \backslash f(N) \subset M \backslash f(N)$ so that $(N \backslash L) \cap f^{-1}((\partial N) \backslash L) \subset N \cap$ $M \backslash N=\varnothing$. This completes the proof of theorem 4.5 .

\section{Index pairs}

Definition 5.1. Let $S$ be an isolated invariant set for a dynamical system $f$ on a space $M$. An index pair for $(S, f)$ is a compact pair $(N, L)$ in $M$ such that $N \backslash L$ is an isolating neighbourhood for $(S, f)$ and the semidynamical system $f_{\#}$ on $N / L$ induced by $f$ is continuous.

We remark that in view of theorem 4.2 this definition agrees with the standard definition (see [1], [2], [9] and [11]) in the continuous case. Our goal in this section is to prove that any isolated invariant set admits an index pair. We shall in fact prove somewhat more; namely that there exist index pairs which are stable (i.e. remain index pairs) under suitable perturbations of the dynamical system $f$.

THEOREM 5.2. Let $V$ be an isolating neighbourhood for $(S, f)$. Then there exists $a$ Lyapunov function for $(S, f)$ on $V$, i.e. a smooth function $\Phi: V \rightarrow \mathbf{R}$ such that

$$
\begin{gathered}
\Phi\left(f^{t}(x)\right)<\Phi(x) \quad \text { if } f^{[0, t]}(x) \subset V \backslash S \text { and } t>0 ; \\
\Phi(x)=0 \quad \text { for } x \in S .
\end{gathered}
$$

Proof. We first consider the discrete time case: $T=Z$. Note that in this case the conclusion of the theorem may be more simply expressed as $\Phi(x)=0$ if $x \in S$ and $\Phi(f(x))<\Phi(x)$ if $x, f(x) \in V \backslash S$.

Step 1. Choose $x_{0} \in(V \backslash S) \cap f^{-1}(V)$; we will define $\Phi_{0}: V \rightarrow \mathbf{R}$ such that $\Phi_{0} \mid S=0$, $\Phi_{0}(f(x)) \leq \Phi_{0}(x)$ for $x \in V \cap f^{-1}(V)$, and $\Phi_{0}\left(f\left(x_{0}\right)\right)<\Phi_{0}\left(x_{0}\right)$. By the definition of isolating neighbourhood the orbit of $x_{0}$ must leave cl $(V)$; choose $q$ so that $f^{q}\left(x_{0}\right) \in$ $M \backslash \mathrm{cl}(V)$. Now choose a neighbourhood $U$ of $x_{0}$ so small that $f^{q}(U) \cap \operatorname{cl}(V)=\varnothing$; i.e.

$$
f^{-q}(\operatorname{cl}(V)) \cap U=\varnothing .
$$

Now choose a smooth function $\phi: M \rightarrow[0,1]$ supported in $U$ with $\phi\left(x_{0}\right) \neq 0$ so that

$$
\phi\left(f^{-q}(x)\right)=0
$$

for $x \in \operatorname{cl}(V)$. Define $\Phi_{0}: V \rightarrow \mathbf{R}$ by the formula

$$
\Phi_{0}(x)=-\sum_{t=1}^{q-1} \phi\left(f^{-t}(x)\right)=-\sum_{t=1}^{q} \phi\left(f^{-t}(x)\right)
$$

if $q$ is positive and by

$$
\Phi_{0}(x)=\sum_{t=0}^{-q-1} \phi\left(f^{t}(x)\right)=\sum_{t=0}^{-q} \phi\left(f^{t}(x)\right)
$$

if $q$ is negative. Then

$$
\Phi_{0}(f(x))=\Phi_{0}(x)-\phi(x)
$$

which proves the desired inequalities. (Note that changing $f$ outside $\mathrm{cl}(V)$ could change $\Phi_{0}$. In the smooth case $\phi$ and hence $\Phi_{0}$ may be chosen as smooth as $f$.) 
Step 2. As $M$ is separable, we may find functions $\Phi_{n}: M \rightarrow \mathbf{R}$ as in step 1 so that the open sets $\left\{x \in M: \Phi_{n}(f(x))<\Phi_{n}(x)\right\}$ cover $(V S) \cap f^{-1}(V)$. We then define $\Phi$ by an infinite series

$$
\Phi(x)=\sum_{n} c_{n} \Phi_{n}(x)
$$

where the coefficients $c_{n}$ are chosen positive and tending to zero so rapidly that the series converges uniformly. (If it is desired that $\Phi$ be of class $C^{r}$, choose the coefficients so that the derivatives of order $\leq r$ converge uniformly as well.)

The continuous time case is quite similar. Step 2 is exactly the same; for step 1 we replace the sum by an integral:

$$
\Phi_{0}(x)=-\int_{-q}^{0} \phi\left(f^{t}(x)\right) d t
$$

As before we obtain an equation

$$
\Phi\left(f^{s}(x)\right)=\Phi(x)-\int_{0}^{s} \phi\left(f^{t}(x)\right) d t
$$

when $f^{[0, s]}(x) \subset V$, which (as $\phi$ is non-negative) implies that $\Phi$ is non-increasing on orbit segments in $V$ and strictly decreasing on the support of $\phi$. This completes the proof of 5.2 .

TheOREM 5.3. Assume the discrete time case: $T=Z$. Let $V$ be an isolating neighbourhood for $(S, f)$ so that $S=I(V, f)$ the maximal invariant set in $V$. Then there exist a neighbourhood $\mathscr{F}$ of $f$ in $\operatorname{Diff}(M)\left(C^{0}\right.$ topology) and a compact pair $(N, L)$ which is an index pair for $(I(V, g), g)$ for any $g \in \mathscr{F}$. One can choose $(N, L)$ so that $N / L$ has the homotopy type of a polyhedron.

Proof. Let $\Phi: V \rightarrow \mathbf{R}$ be a Lyapunov function as in theorem 5.2. By proposition 3.1 we may shrink $V$ if necessary and assume that $\Phi \circ f$ and $\Phi \circ f^{-1}$ are both defined on $V$. Choose $\varepsilon>0$ so small that

$$
x \in \partial V \Rightarrow \Phi(x)<\Phi\left(f^{-1}(x)\right)-2 \varepsilon
$$

and define $N \subset V$ by

$$
N=\left\{x \in V:-\varepsilon \leq \Phi(x) \leq \Phi\left(f^{-1}(x)\right) \leq \varepsilon\right\} .
$$

As the inequalities defining $N$ cannot hold for $x \in \partial V$, we have

$$
\partial N \subset \Phi^{-1}(-\varepsilon) \cup f\left(\Phi^{-1}(\varepsilon)\right) ;
$$

we take

$$
L=N \cap \Phi^{-1}(-\varepsilon) .
$$

Then $N \cap \Phi^{-1}(\varepsilon)=\varnothing$ and $f(L) \cap N=\varnothing$ so that there exists a neighbourhood $\mathscr{F}$ of $f$ in Diff $(M)$ with $g(N) \cap f\left(\Phi^{-1}(\varepsilon)\right)=\varnothing$ and $g(L) \cap N=\varnothing$ for $g \in \mathscr{F}$. Hence

$$
(\partial N) \cap g(N) \subset L \subset N \backslash g^{-1}(N)
$$

for $g \in \mathscr{F}$. From theorem 4.5 it follows that $g_{\#}$ is continuous.

From $S \subset$ int $(V) \cap \Phi^{-1}(0)$ we obtain $S \subset$ int $(N) \subset V$. From $S \subset$ int $(N)$ we obtain $S \subset$ int $\left(f^{-1}(N)\right)$ so that $S \subset N \backslash L \subset V$. Thus $N \backslash L$ is an isolating neighbourhood for 


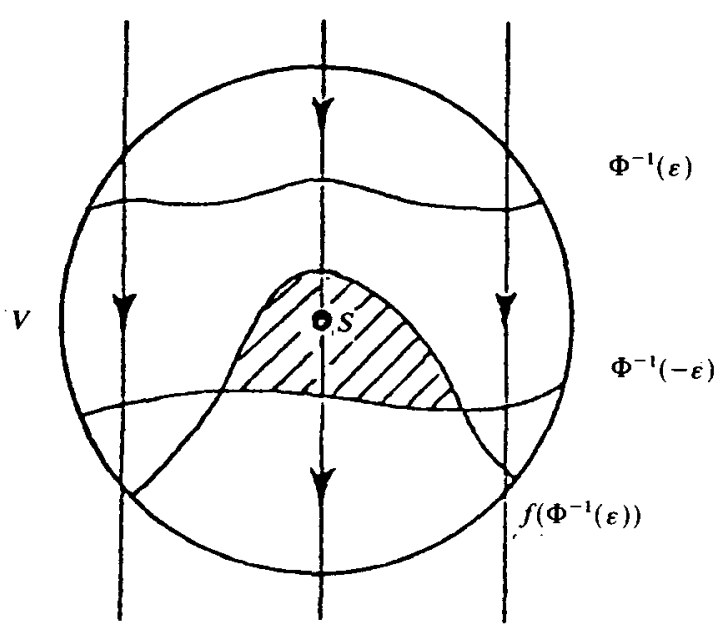

$(S, f)=(I(V, f), f)$. By proposition 3.1 we have that $N \backslash L$ is an isolating neighbourhood for $(I(V, g), g)$ for $g$ sufficiently near $f$. We note that we can also choose $\varepsilon$ so that $-\varepsilon$ is a regular value of $\Phi$ and $\varepsilon$ is a regular value of $\Psi=\Phi \circ f^{-1}$ (Sard's theorem [5]) and then perturb $\Phi$ and $\Psi$ so that $\Phi^{-1}(-\varepsilon)$ and $\Psi^{-1}(\varepsilon)$ are transverse. Then by lemma $2.8 \mathrm{~N} / L$ is a homotopy polyhedron. This completes the proof of theorem 5.3.

Example. A good example to keep in mind is the following. Take $M=\mathbf{R}^{2}$ with $f^{t}(x, y)=\left(2^{t} x, 2^{-t} y\right)$. Take $V=[-1,1] \times[-1,1]$ an isolating neighbourhood for the hyperbolic fixed point $S=\{(0,0)\}$. Take the Lyapunov function $\Phi(x, y)=y^{2}-x^{2}$. Then $N$ is bounded by two hyperbolas with four points of intersection (inside $V$ if $\varepsilon$ is small enough) and $L$ is a portion of one of them. This same example illustrates both the discrete time case and the continuous time case which follows.

THEOREM 5.4. Assume the continuous time case: $T=\mathbf{R}$. Let Vbe an isolating neighbourhood for $(S, f)$ so that $S=I(V, f)$ the maximal invariant set in $V$. Let $v \in \mathscr{X}(M)$ be the infinitesimal generator of $f$. Then there exist a neighbourhood $\mathscr{F}$ of $v$ in $\mathscr{Z}(M)$ ( $C^{0}$ topology) and a compact pair $(N, L)$ which is an index pair for $(I(V, g), g)$ for any flow $g$ whose generator $w$ lies in $\mathscr{F}$. One can choose $(N, L)$ so that $N / L$ has the homotopy type of a polyhedron.

Proof. As in the discrete case we take

$$
N=\left\{x \in V:-\varepsilon \leq \Phi(x) \leq \Phi\left(f^{-\delta}(x)\right) \leq \varepsilon\right\},
$$

where $\Phi$ is a Lyapunov function defined on a neighbourhood of the closure of $V, \delta>0$ is small enough that $f^{[-\delta, \delta]}(x)$ lies in the domain of $\Phi$ for $x \in V_{n}$ and $\varepsilon>0$ is small enough so that

$$
\partial N \subset \Phi^{-1}(-\varepsilon) \cup f^{\delta}\left(\Phi^{-1}(\varepsilon)\right) .
$$

Choose a neighbourhood $\mathscr{F}$ of $v$ in $\mathscr{X}(M)$ so that

$$
d \Phi(x) w(x)<0, \quad d\left(\Phi \circ f^{-\delta}\right)(x) w(x)<0
$$

SOS XE AN ANB WE 
constructed above, $x \in V \backslash S$.) We take

$$
L=\Phi^{-1}(-\varepsilon) \cap N
$$

Choose $w \in \mathscr{F}$ and let $g$ denote the flow generated by $w$. If $x \in L$, then $g^{t}(x) \notin N$ for small positive $t$; while if $x \in(\partial N) \backslash L$, then $g^{\prime}(x) \in$ int $(N)$ for small positive $t$. Thus $(N, L)$ satisfies the conditions of theorem 4.2 and so is an index pair as required. We show $N / L$ is a homotopy polyhedron as before.

We remark that theorem 5.4 cannot possibly hold if one requires only that the flow $g$ be close to $f$ in the $C^{0}$ sense (as opposed to their generators being close). The reason is that the condition that $L$ be positively invariant relative to $N$ can always be violated by a small perturbation of the flow.

COROLlary 5.5. Let $\left\{\left(S_{\lambda}, f_{\lambda}\right): \lambda \in J\right\}$ be a continuation of isolated invariant sets. Then there is a covering $J_{j}(j=1,2, \ldots, n)$ of the unit interval $J$ and pairs $\left(N_{j}, L_{j}\right)$ $(j=1,2, \ldots, n)$ such that $\left(N_{j}, L_{j}\right)$ is an index pair for $\left(S_{\lambda}, f_{\lambda}\right)$ for $\lambda \in J_{j}$.

\section{The index category}

In this section we fix an isolated invariant set $S$ for a dynamical system $f$ on $M$ and choose an indexing $\left\{\left(N_{\alpha}, L_{\alpha}\right)\right\}_{\alpha}$ of all the index pairs for $(S, f)$.

Definition 6.1. Let $\left(N_{\alpha}, L_{\alpha}\right)$ and $\left(N_{\beta}, L_{\beta}\right)$ be index pairs for $(S, f)$ and $t \in T^{+}$. The morphism from $N_{\alpha} / L_{\alpha}$ to $N_{\beta} / L_{\beta}$ induced by the dynamical system fat time $t$ is the map

$$
f_{\beta \alpha}^{t}: N_{\alpha} / L_{\alpha} \rightarrow N_{\beta} / L_{\beta}
$$

defined by

$$
f_{\beta \alpha}^{t}(x)= \begin{cases}f^{\prime}(x) & \text { if } x \in C_{\alpha \beta}^{t}, \\ * & \text { otherwise, }\end{cases}
$$

where the set $C_{\alpha \beta}^{\prime}$ is the subset of $N_{\alpha} \backslash L_{\alpha}$ defined by

$$
x \in C_{\alpha \beta}^{t} \Leftrightarrow\left\{\begin{array}{l}
f^{[0,2 t / 3]}(x) \subset N_{\alpha} \backslash L_{\alpha} \text { and } \\
f^{[t / 3, t]}(x) \subset N_{\beta} \backslash L_{\beta} .
\end{array}\right.
$$

When $\alpha=\beta$ we abbreviate $f_{\alpha \alpha}^{t}$ to $f_{\alpha}^{t}$ :

$$
f_{\alpha}^{t}=f_{\alpha \alpha}^{t}: N_{\alpha} / L_{\alpha} \rightarrow N_{\alpha} / L_{\alpha}
$$

LEMMA 6.2. There is a function which assigns to each pair $(\alpha, \beta)$ of index pairs for $(S, f)$ a number $t_{\alpha \beta}$ having the following properties:

$$
\begin{gathered}
t_{\alpha \alpha}=0, \quad t_{\alpha \beta}=t_{\beta \alpha}, \quad t_{\alpha \gamma} \leq t_{\alpha \beta}+t_{\beta \gamma}, \\
t \geq t_{\alpha \beta} / 3, \quad f^{[a, b]}(x) \subset N_{\alpha} \backslash L_{\alpha} \Rightarrow f^{[a+t, b-t]}(x) \subset N_{\beta} \backslash L_{\beta} .
\end{gathered}
$$

Proof. To define $t_{\alpha \beta}$, note that by proposition 3.1 we have

$$
f^{[-t, t]}(x) \subset N_{\alpha} \backslash L_{\alpha} \Rightarrow x \in N_{\beta} \backslash L_{\beta}
$$

and

$$
f^{[-t, t]}(x) \subset N_{\beta} \backslash L_{\beta} \Rightarrow x \in N_{\alpha} \backslash L_{\alpha}
$$

for sufficiently large $t \geq 0$; we denote by $u_{\alpha \beta}$ the infimum of all such $t$ and take $t_{\alpha \beta}=3 u_{\alpha \beta}$ if $\alpha \neq \beta$ with $t_{\alpha \alpha}=0$. To show $t_{\alpha \gamma} \leq t_{\alpha \beta}+t_{\beta \gamma}$, it suffices to show $u_{\alpha \gamma} \leq$ 
$u_{\alpha \beta}+u_{\beta \gamma}$. For this we must show that if $t>u_{\alpha \beta}, s>u_{\beta \gamma}$ and $f^{[a, b]}(x) \subset N_{\alpha} \backslash L_{\alpha}$, then $f^{[a+t+s, b-t-s]}(x) \subset N_{\gamma} \backslash L_{\gamma}$. This follows as $f^{[a, b]}(x) \subset N_{\alpha} \backslash L_{\alpha}$ implies $f^{[a+t, b-t]}(x) \subset$ $N_{\beta} \backslash L_{\beta}$ which implies $f^{[a+t+s, b-t-s]}(x) \subset N_{\gamma} \backslash L_{\gamma}$. This proves the lemma.

THEOREM 6.3. (i) The morphism $f_{\beta \alpha}^{t}$ is continuous for $t>t_{\alpha \beta}$.

(ii) The morphisms $f_{\alpha}^{t}$ form the semidynamical system on $N_{\alpha} / L_{\alpha}$ induced by the dynamical system $f$; i.e.

$$
f_{\alpha}^{t}=f_{\#}^{t}: N_{\alpha} / L_{\alpha} \rightarrow N_{\alpha} / L_{\alpha}
$$

(iii) If $t>t_{\alpha \beta}$ and $s>t_{\beta \gamma}$, then $s+t>t_{\alpha \gamma}$ and

$$
f_{\gamma \alpha}^{s+t}=f_{\gamma \beta}^{s} \circ f_{\beta \alpha}^{t} \text {. }
$$

Definition 6.4. These conditions say that there is a category whose objects are the spaces $N_{\alpha} / L_{\alpha}$, where $\left(N_{\alpha}, L_{\alpha}\right)$ is an index pair for $(S, f)$, and whose morphisms are the morphisms $f_{\beta \alpha}^{t}: N_{\alpha} / L_{\alpha} \rightarrow N_{\beta} / L_{\beta}$ induced by the dynamical system $f$ at times $t>t_{\alpha \beta}$. We call this category the index category of $(S, f)$. It contains as morphisms the elements $f_{\alpha}^{t}: N_{\alpha} / L_{\alpha} \rightarrow N_{\alpha} / L_{\alpha}$ of the semigroup induced by $f$.

Proof of 6.3. We consider the discrete time case: $T=Z$; the continuous time case is similar (see [9]). We prove that $f_{\beta \alpha}^{t}$ is continuous at $x_{0} \in N_{\alpha} / L_{\alpha}$ via a case analysis. Case 1. $f^{[0,2 t / 3]}\left(x_{0}\right) \cap\left(M \backslash N_{\alpha}\right) \neq \varnothing$ or $f^{[t / 3, t]}\left(x_{0}\right) \cap\left(M \backslash N_{\beta}\right) \neq \varnothing$. Then these intersections continue to be non-empty for $x$ near $x_{0}$, so $f_{\beta \alpha}^{\prime}(x)=*$ for such $x$.

Case 2. $f^{\prime}\left(x_{0}\right) \in L_{\beta}$. Then $f^{t}(x)$ is near $L_{\beta}$ for $x$ near $x_{0}$, so $f_{\beta \alpha}^{t}(x)$ is near $*$ for such $x$.

Case 3, $f^{[0,2 t / 3]}\left(x_{0}\right) \subset N_{\alpha}, f^{[t / 3, t]}\left(x_{0}\right) \subset N_{\beta}, f^{\prime}\left(x_{0}\right) \in N_{\beta} \backslash L_{\beta}$, but $f^{s}\left(x_{0}\right) \in L_{\beta}$ for some $s \in[t / 3, t]$. Take the largest such $s$; then by condition (A0) of theorem 4.3 we obtain that $f^{s+1}(x) \notin N_{\beta}$ if $x$ is near $x_{0}$ and $f^{s}(x) \in N_{\beta} \backslash L_{\beta}$, so that $f_{\beta \alpha}^{t}(x)=*$ for $x$ near $x_{0}$. Case 4. $f^{[0,2 t / 3]}\left(x_{0}\right) \subset N_{\alpha}, f^{[t / 3, t]}\left(x_{0}\right) \subset N_{\beta} \backslash L_{\beta}$ and $f^{s}\left(x_{0}\right) \in L_{\alpha}$ for some $s \in[0,2 t / 3]$. Suppose that $s$ is the largest integer with this property. We obtain from lemma 6.2 and $t>t_{\alpha \beta}$ that $s<t-t_{\alpha \beta} / 3$ and $f^{s+1}\left(x_{0}\right) \in N_{\alpha} \backslash L_{\alpha}$. Hence it follows from condition (A0) of theorem 4.3 that $f^{s+1}(x) \notin N_{\alpha}$ if $x$ is near $x_{0}$ and $f^{s}(x) \in N_{\alpha} \backslash L_{\alpha}$. Since $t_{\alpha \beta} / 3$ is an integer, we obtain $s+1 \leq t-t_{\alpha \beta} / 3$ and it follows again from lemma 6.2 that $x \notin C_{\alpha \beta}^{t}$ if $x \in N_{\alpha} \backslash L_{\alpha}$ is near $x_{0}$. Hence $f_{\beta \alpha}^{t}(x)=*$ for $x$ near $x_{0}$.

Case 5. $f_{\beta \alpha}^{[0,2 t / 3]}\left(x_{0}\right) \subset N_{\alpha} \backslash L_{\alpha}$ and $f_{\beta \alpha}^{[t / 3, t]}\left(x_{0}\right) \subset N_{\beta} \backslash L_{\beta}$. It follows from lemma 6.2 that $f^{s}\left(x_{0}\right) \in N_{\alpha} \backslash L_{\alpha}$ for $0 \leq s \leq t-t_{\alpha \beta} / 3$. By condition (B0) of theorem 4.3 this implies that $f^{s}(x) \in N_{\alpha} \backslash L_{\alpha}$ for $0 \leq s \leq t-t_{\alpha \beta} / 3$ for $x$ near $x_{0}$. Now it follows again from lemma 6.2 that $f^{s}(x) \in N_{\beta} \backslash L_{\beta}$ for $t_{\alpha \beta} / 3 \leq s \leq t$ for $x$ near $x_{0}$. We conclude that a neighbourhood of $x_{0}$ in $N_{\alpha} \backslash L_{\alpha}$ is contained in $C_{\alpha \beta}^{\prime}$, so that the continuity of $f_{\beta \alpha}^{t}$ at $x_{0}$ follows from the continuity of $f^{t}$.

This completes the proof of (i).

Assertion (ii) is obvious: the condition $x \in C_{\alpha \alpha}^{\prime}$ means that $f^{[0, t]}(x) \subset N_{\alpha} \backslash L_{\alpha}$, so that $f_{\alpha \alpha}=f_{\#}$.

To prove (iii) we must show that $x \in C_{\alpha \gamma}^{s+t}$ if and only if $x \in C_{\alpha \beta}^{\prime}$ and $f^{t}(x) \in C_{\beta \gamma}^{s}$. Assume $x \in C_{\alpha \beta}^{\prime}$ and $f^{t}(x) \in C_{\beta \gamma}^{s}$. Reason as follows:

$$
f^{[0,2 t / 3]}(x) \subset N_{\alpha} \backslash L_{\alpha}, \quad f^{[t / 3, t]}(x) \subset N_{\beta} \backslash L_{\beta}
$$


(as $x \in C_{\alpha \beta}^{\prime}$ ) and

$$
f^{[t, t+2 s / 3]}(x) \subset N_{\beta} \backslash L_{\beta}, \quad f^{[t+s / 3, t+s]}(x) \subset N_{\gamma} \backslash L_{\gamma},
$$

(as $f^{t}(x) \in C_{\beta \gamma}^{s}$ ). Combining gives

$$
f^{[t / 3, i+2 s / 3]}(x) \subset N_{\beta} \backslash L_{\beta},
$$

so (as $s>t_{\beta \gamma}$ and $t>t_{\alpha \beta}$ )

$$
f^{[t / 3+s / 3, t+s / 3]}(x) \subset N_{\gamma} \backslash L_{\gamma}, \quad f^{[2 t / 3,2 t / 3+2 s / 3]}(x) \subset N_{\alpha} \backslash L_{\alpha} .
$$

Combining gives

$$
f^{[(t+s) / 3, t+s]}(x) \subset N_{\gamma} \backslash L_{\gamma}, \quad f^{[0,2(t+s) / 3]}(x) \subset N_{\alpha} \backslash L_{\alpha} .
$$

Therefore $x \in C_{\alpha \gamma}^{s+t}$.

Conversely assume $x \in C_{\alpha \gamma}^{s+t}$, i.e. (3). Then (as $s>t_{\beta \gamma}$ and $t>t_{\alpha \beta}$ )

$$
f^{[1 / 3+2 s / 3, t+2 s / 3]}(x) \subset N_{\beta} \backslash L_{\beta}, \quad f^{[t / 3, t / 3+2 s / 3]}(x) \subset N_{\beta} \backslash L_{\beta},
$$

i.e.

$$
f^{[t / 3, t+2 s / 3]}(x) \subset N_{\beta} \backslash L_{\beta},
$$

which (together with (3)) gives (1) and (2). Therefore $x \in C_{\alpha \beta}^{t}$ and $f^{t}(x) \in C_{\beta \gamma}^{s}$. This completes the proof of (iii) and thus of theorem 6.3.

\section{The unstable manifold}

In this section time is either continuous or discrete. Let $S$ be an isolated invariant set for a dynamical system $f$ on $M$.

Definition 7.1. The unstable manifold $W^{u}(S, f)$ of the invariant set $S$ is the set of all points of $M$ whose orbit tends to $S$ in backwards time:

$$
W^{\mathrm{u}}(S, f)=\left\{x \in M: \lim _{t \rightarrow \infty} d\left(f^{-t}(x), S\right)=0\right\}
$$

where $d$ denotes any metric giving the topology of $M$.

Proposition 7.2. If $V$ is an isolating neighbourhood for $(S, f)$, then $x \in W^{\mathrm{u}}(S, f)$ if and only if $f^{-t}(x) \in V$ for all sufficiently large $t$; i.e.

$$
W^{\mathrm{u}}(S, f)=\bigcup_{t_{0}>0} \bigcap_{t>t_{0}} f^{\prime}(V)
$$

Fix an index pair $\left(N_{\alpha}, L_{\alpha}\right)$ for $(S, f)$. We will define an inverse system $\left(N_{\alpha} / L_{\alpha}, f_{\alpha}\right)$ indexed by $T^{+}$. All the spaces are the same:

$$
\left(N_{\alpha} / L_{\alpha}\right)_{s}=N_{\alpha} / L_{\alpha}
$$

for $s \in T^{+}$. The bonding maps are the elements of the semigroup

$$
f_{\alpha}^{t}:\left(N_{\alpha} / L_{\alpha}\right)_{s+t} \rightarrow\left(N_{\alpha} / L_{\alpha}\right)_{s}
$$

induced by $f$. We denote by $W_{\alpha}^{\#}$ the inverse limit

$$
W_{\alpha}^{\#}=\lim \left(N_{\alpha} / L_{\alpha}, f_{\alpha}\right) \text {. }
$$

A point $\xi \in W_{\alpha}^{\#}$ is a map $\xi: T^{+} \rightarrow N_{\alpha} / L_{\alpha}$ satisfying

$$
\xi(s)=f_{\alpha}^{t}(\xi(t+s))
$$

for $t, s \in T^{+}$. The base point of $W_{\alpha}^{*}$ is the constant map whose value is the base 
point $* \in N_{\alpha} / L_{\alpha}$; we denote by $W_{\alpha}$ the complement of the base point:

$$
W_{\alpha}=W_{\alpha}^{\#} \backslash\{*\} \text {. }
$$

Thus a typical point $\xi \in W_{\alpha}$ is a map $\xi: T^{+} \rightarrow N_{\alpha} / L_{\alpha}$ either of form

$$
\xi(t)=f^{-t}(x)
$$

for all $t \in T^{+}$and some $x \in N_{\alpha} \backslash L_{\alpha}$ or of form

$$
\xi(t)= \begin{cases}* & \text { if } t \leq \tau \\ f^{r-t}(x) \in N_{\alpha} \backslash L_{\alpha} & \text { if } \tau<t\end{cases}
$$

for all $t \in T^{+}$, some $\tau=\tau(\xi) \in T^{+}$and some $x=\xi(\tau) \in N_{\alpha}$. (This because if $f_{\alpha}^{\prime}(x)=*$ for some $t$, then this continues to hold for all larger $t$.) Define

$$
\iota_{\alpha}: W_{\alpha} \rightarrow M
$$

by

$$
\iota_{\alpha}(\xi)=f^{t}(\xi(t))
$$

(The result is independent of the choice of $t>\tau(\xi)$ ).

Note that there is a dynamical system

$$
\sigma_{\alpha}^{t}: W_{\alpha}^{\#} \rightarrow W_{\alpha}^{\#}
$$

$(t \in T)$ so that $\iota_{\alpha}: W_{\alpha} \rightarrow M$ intertwines $\sigma_{\alpha}$ and $f$ :

$$
\iota_{\alpha} \circ \sigma_{\alpha}^{t}=f^{t} \circ \iota_{\alpha} \text {. }
$$

Indeed $\sigma_{\alpha}$ is the (two-sided) shift; it is defined by

$$
\sigma_{\alpha}^{t}(\xi)(s)=\xi(s-t)
$$

for large $s$ (this determines it for all $s$ ).

THEQREM 7,3. The map $v_{\alpha}$ is a continuous injection whose image is the unstable manifold $W^{\mathrm{u}}(S, f)$ of the isolated invariant set $S$ :

$$
\iota_{\alpha}\left(W_{\alpha}\right)=W^{\mathrm{u}}(S, f) \text {. }
$$

If $\left(N_{\beta}, L_{\beta}\right)$ is another index pair for $(S, f)$, then the bijective map

$$
\iota_{\beta}^{-1} \circ \iota_{\alpha}: W_{\alpha} \rightarrow W_{\beta}
$$

is a homeomorphism.

Definition 7.4. Thus the topology on $W^{\mathrm{u}}(S, f)$ which makes $\iota_{\alpha}$ a homeomorphism is independent of the choice of the index pair $\left(N_{\alpha}, L_{\alpha}\right)$; it is called the intrinsic topology on $W^{\mathrm{u}}(S, f)$. The topology which $W^{\mathrm{u}}(S, f)$ inherits as a subset of $M$ will be called the extrinsic topology on $W^{\mathrm{u}}(S, f)$. We denote by $W^{*}(S, f)$ the one-point compactification of $W^{\mathrm{u}}(S, f)$ in its intrinsic topology, so by definition $\iota_{\alpha}$ extends to a homeomorphism (denoted by the same symbol)

$$
\iota_{\alpha}: W_{\alpha}^{\#} \rightarrow W^{\#}(S, f) \text {. }
$$

Proof of theorem 7.3. The idea of the definitions just given is that a point $x \in W^{\mathrm{u}}(S, f)$ will be identified with its backwards orbit $\xi(t)=f^{-1}(x)$ which will lie in $N \backslash L$ for large $t$. Of course any point in the orbit determines the whole orbit. We modify the first part of $\xi$ in the only way we can (i.e. setting $\xi(t)=*$ for small $t$ ) in order to get a point of the inverse limit space. 
Now we proceed to the details. Since $\iota_{\alpha}(\xi)=f^{t}(\xi(t))$ for large $t$, it follows immediately that $\iota_{\alpha}$ is continuous and injective. Since $f^{-t}\left(\iota_{\alpha}(\xi)\right)=\xi(t) \in N_{\alpha} \backslash L_{\alpha}$ for large $t$, it follows by proposition 7.2 that $\iota_{\alpha}(\xi) \in W^{\mathrm{u}}(S, f)$. Conversely, if $x \in W^{\mathrm{u}}(S, f)$, then $\xi \in W_{\alpha}$ defined by $\xi(t)=f^{-t}(x)$ for large $t$ maps to $x$ under $\iota_{\alpha}$. This shows that $\iota_{\alpha}$ is a continuous bijection.

We shall describe the map $\iota_{\beta}^{-1} \circ \iota_{\alpha}$ in another way, using the index category, which makes its continuity obvious. Given $q, t \in T^{+}$with $t$ sufficiently large, we have the morphism

$$
f_{\beta \alpha}^{t}:\left(N_{\alpha} / L_{\alpha}\right)_{q+t} \rightarrow\left(N_{\beta} / L_{\beta}\right)_{q}
$$

By theorem 6.3

$$
f_{\beta \alpha}^{t} \circ f_{\alpha}^{r}=f_{\beta}^{r} \circ f_{\beta \alpha}^{t},
$$

i.e. we have a morphism of inverse systems and hence an induced map on the inverse limits:

$$
\iota_{\beta \alpha}: W_{\alpha}^{\#}=\lim \left(N_{\alpha} / L_{\alpha}, f_{\alpha}\right) \rightarrow W_{\beta}^{\#}=\lim \left(N_{\beta} / L_{\beta}, f_{\beta}\right)
$$

(The definition is independent of the choice of $t>t_{\alpha \beta}$.) By theorem 6.3 again $\iota_{\gamma \alpha}=\iota_{\gamma \beta} \circ \iota_{\beta \alpha}$ and $\iota_{\alpha \alpha}=$ id so that $\iota_{\beta \alpha}$ is a homeomorphism with inverse $\iota_{\alpha \beta}$.

All that remains is to check that $\iota_{\beta \alpha}=\iota_{\beta}^{-1} \circ \iota_{\alpha}$. For that choose $\xi \in W_{\alpha}^{\#}$ and put $\eta=\iota_{\beta}^{-1} \circ \iota_{\alpha}(\xi)$. Then $\iota_{\beta}(\eta)=\iota_{\alpha}(\xi)=f^{r}(\eta(r))=f^{r}(\xi(r))$ for large $r$ and in particular $\eta(r)=\xi(r) \in\left(N_{\alpha} \backslash L_{\alpha}\right) \cap\left(N_{\beta} \backslash L_{\beta}\right)$ for all sufficiently large $r$. Then clearly $f_{\beta \alpha}^{t}(\xi(r+t))=\eta(r)$ for fixed $t>t_{\alpha \beta}$ and large enough $r$, i.e. $\iota_{\beta \alpha}(\xi)=\eta$. This proves theorem 7.3.

If $S$ is a hyperbolic fixed point for $f$, then it is an isolated invariant set and $W^{\mathrm{u}}(S, f)$ in its intrinsic topology is homeomorphic to Euclidean space so $W^{\#}(S, f)$ is homeomorphic to a sphere. If, however, the stable and unstable manifolds of $S$ intersect transversally, as in the Smale horseshoe, then $W^{\mathrm{u}}(S, f)$ will not be embedded and so the intrinsic and extrinsic topologies will not be the same.

Proposition 7.5. If $(S, f)$ admits an index pair $(N, L)$ with both $N$ and $L$ positively invariant by $f$ (i.e. $f^{\prime}(N) \subset N$ and $f^{t}(L) \subset L$ for $t \in T^{+}$), then the intrinsic topology and the extrinsic topology on $W^{\mathrm{u}}(S, f)$ agree.

Proof. Let $W^{\#}=\lim (N / L, f)$ and $W=W^{\#} \backslash\{*\}$. We must show that the continuous bijection $\iota: W \rightarrow W^{\mathrm{u}}(S, f)$ is a homeomorphism. Choose a sequence $\xi_{n} \in W$ with $x_{n}=\iota\left(\xi_{n}\right)$ converging to a point $x=\iota(\xi) \in W^{\mathrm{u}}(S, f)$; we must have that $\xi_{n}$ converges to $\xi$. Applying a suitable shift $\sigma^{t}$, we may assume without loss of generality that $x \in$ int $(N \backslash L)$ so that $x=\xi(0)$. For $n$ sufficiently large we have $x_{n} \in$ int $(N \backslash L)$ as well; it suffices to show $x_{n}=\xi_{n}(0)$. (If $\xi_{n}(0) \neq *$, then $\xi_{n}(t)=f^{-t}\left(\xi_{n}(0)\right) \in N \backslash L$ for all $t$ so that the convergence of $\xi_{n}$ to $\xi$ is assured by the continuity of $f^{-t}$.) Now for large $t$ we have $x_{n}=f^{t}\left(\xi_{n}(t)\right)$ (by the definition of $\iota$ ) and $\xi_{n}(t) \in N \backslash L$ (as $\xi \in W$ ). Since $\xi_{n}(0)=f_{*}^{t}\left(\xi_{n}(t)\right)$ for all $t$, the only way we can have $x_{n} \neq \xi_{n}(0)$ is if $\xi_{n}(0)=*$, and this means that for some $s \in T^{+}$we have $f^{-s}\left(x_{n}\right) \notin N \backslash L$. But we cannot have $f^{-s}\left(x_{n}\right) \notin N$ as $N$ is positively invariant and $f^{-t}\left(x_{n}\right) \in N \backslash L$ for large $t$, and we cannot have $f^{-s}\left(x_{n}\right) \in L$ for then (since $L$ is positively invariant) we would have $x_{n} \in L$ contradicting $x_{n} \in$ int $(N \backslash L)$. This completes the proof. 
8. The homotopy (Conley) index

In this section we consider only the continuous time case: $T=\mathbf{R}$.

THEOREM 8.1. Let $S$ be an isolated invariant set for the flow fon $M$ and let $\left(N_{\alpha}, L_{\alpha}\right)$ and $\left(N_{\beta}, L_{\beta}\right)$ be index pairs for $(S, f)$. Then the spaces $N_{\alpha} / L_{\alpha}$ and $N_{\beta} / L_{\beta}$ are homotopy equivalent:

$$
\left[N_{\alpha} / L_{\alpha}\right]=\left[N_{\beta} / L_{\beta}\right] \text {. }
$$

Proof. For $t>t_{\alpha \beta}$ the map $f_{\beta \alpha}^{t}: N_{\alpha} / L_{\alpha} \rightarrow N_{\beta} / L_{\beta}$ is a homotopy equivalence with homotopy inverse $f_{\alpha \beta}^{t}$. This is because $f_{\beta \alpha}^{t} \circ f_{\alpha \beta}^{t}=f_{\beta}^{2 t}$ and $f_{\alpha \beta}^{t} \circ f_{\beta \alpha}^{t}=f_{\alpha}^{2 t}$, and $f_{\beta}^{2 t}$ and $f_{\alpha}^{2 t}$ are homotopic to the identity (the homotopy $s \mapsto f_{\alpha}^{s}$ with $0 \leq s \leq 2 t$ connects the identity to $f_{\alpha}^{2 t}$ ).

Definition 8.2. The common homotopy type is called the homotopy index or the Conley index of $(S, f)$ and is denoted by $h(S, f)$. Thus

$$
h(S, f)=[N / L]
$$

for any index pair $(N, L)$ for $(S, f)$.

THEOREM 8.3. The homotopy index is a continuation invariant; i.e. if $\left(S_{0}, f_{0}\right)$ is a continuation of $\left(S_{1}, f_{1}\right)$, then $h\left(S_{0}, f_{0}\right)=h\left(S_{1}, f_{1}\right)$.

Proof. This follows immediately from corollary 5.5. We remark that this proof assumes the continuation to be $C^{1}$; older proofs are slightly more complicated, but assume only continuity and thus work on an arbitrary metric space (see e.g. [1], [9]).

\section{The shape index}

Definition 9.1. The shape of the topological space $W^{\#}(S, f)$ is called the shape index of $(S, f)$ and denoted by $s(S, f)$ :

$$
s(S, f)=\operatorname{Sh}\left(W^{\#}(S, f)\right)
$$

THEOREM 9.2. The shape index is invariant under continuation. More precisely, if $\left(S_{0}, f_{0}\right)$ is a continuation of $\left(S_{1}, f_{1}\right)$, then

$$
s\left(S_{0}, f_{0}\right)=s\left(S_{1}, f_{1}\right) \text {. }
$$

Proof. This follows immediately from proposition 5.5 and corollary 2.6.

Remark 9.3. Assume the continuous time case: $T=\mathbf{R}$. Then the shape index and the homotopy index contain the same information. More precisely:

(i) If $(N, L)$ is an index pair for $(S, f)$, then $N / L$ and $W^{\#}(S, f)$ have the same shape:

$$
\operatorname{Sh}(N / L)=\operatorname{Sh}\left(W^{\#}(S, f)\right)=s(S, f) .
$$

(Hence $W^{\#}(S, f)$ has the shape of a polyhedron.)

(ii) Two isolated invariant sets have the same shape index if and only if they have the same homotopy index:

$$
s(S, f)=s\left(S^{\prime}, f^{\prime}\right) \Leftrightarrow h(S, f)=h\left(S^{\prime}, f^{\prime}\right) .
$$

(Corollary 2.7 implies (i); (i) and corollary 2.5 imply (ii).) 
Of course, this does not mean that $h(S, f)=\left[W^{*}(S, f)\right]$ in general, although this is true in simple examples. The illuminating example is a flow having the Warsaw circle $S$ as an attractor with an isolating neighbourhood an annulus $N$ on which the flow points in. Then $L=\varnothing$ and $W^{\mathrm{u}}(S, f)=S$ in this case. This invariant set can be continued to an ordinary circle through flows, all of which point into the annulus (thus providing a proof that the Warsaw circle has the shape of a circle). But the Warsaw circle and the circle are not homotopy equivalent (that is the whole point) as the former has a trivial fundamental group.

Acknowledgements. We would like to thank Morton Brown for suggesting that our results had something to do with shape theory and Chris McCord and Sufian Husseini for helpful conversations. We would also like to take this opportunity to eulogize Charley Conley. He was a magnificent teacher, a valued colleague and an original mathematician. We shall miss him immensely.

\section{REFERENCES}

[1] C. C. Conley. Isolated Invariant Sets and the Morse Index. Conf. Board Math. Sci. 38. AMS, Providence, RI (1978).

[2] C. C. Conley \& E. Zehnder. Morse type index theory for flows and periodic solutions for Hamiltonian equations. Commun. Pure Appl. Math. 37 (1984), 207-253.

[3] S. Eilenberg \& N. Steenrod. Foundations of Algebraic Topology. Princeton University Press, Princeton, NJ (1952).

[4] J. Franks. Homology Theory and Dynamical Systems. Conf. Board Math. Sci. 49, AMS, Providence, RI (1982).

[5] M. Hirsch. Differential Topology. Graduate Text in Math. 33. Springer-Verlag, New York (1976).

[6] H. Kurland. The Morse index of an isolated invariant set is a connected simple system. J. Differential Equations 42 (1982), 234-259.

[7] S. Mardešić \& J. Segal. Shape Theory. North-Holland, Amsterdam (1982).

[8] J. Milnor. Morse Theory. Princeton University Press, Princeton, NJ (1963).

[9] D. Salamon. Connected simple systems and the Conley index of isolated invariant sets. Trans. AMS 291 (1985), 1-41.

[10] S. Smale. Differentiable dynamical systems. Bull. AMS 73 (1967), 747-817.

[11] J. Smoller. Shock Waves and Reaction Diffusion Equations. Grund. der Math. Wissen. 258. Springer, New York (1982). 